\title{
Many-Worlds, Self, and Dreams
}

\author{
Lei Ma \\ Huaqiao University, Xiamen, China \\ Email: yuxuanma@hotmail.com
}

How to cite this paper: Ma, L. (2017). ManyWorlds, Self, and Dreams. Open Journal of Philosophy, 7, 353-376.

https://doi.org/10.4236/ojpp.2017.73019

Received: June 29, 2017

Accepted: August 5, 2017

Published: August 8, 2017

Copyright (C 2017 by author and Scientific Research Publishing Inc. This work is licensed under the Creative Commons Attribution International License (CC BY 4.0).

http://creativecommons.org/licenses/by/4.0/

\begin{abstract}
The many-worlds interpretation offers a fundamental explanation to the seemingly bizarre quantum theory; however, it lacks empirical significance. In this work I put forward the concepts of "nousor" and "counterpart", and the notion of self structure scheme, by which I argue that the existence of other parallel worlds could be revealed by dream, and those counterparts in different worlds can exchange information through the special nousor to ensure the oneness of counterparts. Many worlds and all lives there follow the rules of correspondence, convergence, limitation, as well as love and hate. Based on the proposed theory, I analyze not only déjà vu phenomenon, but also actual dreams and sleepwalking, to assist us to understand the concept of self. A large database of humanity's dreams should be constructed for helping predict disruptions both individually and environmentally.
\end{abstract}

\section{Keywords}

Many-Words Theory, Self, Dreams, Sleepwalking, Déjà Vu

\section{Introduction}

In this paper I only make a metaphysical argument that we ought to forgo claiming any epistemic warrant for taking cognitive constructs as representative of the nature of the world in any sense. It is necessary to construct a fanciful inner world in scientific practice, because the legitimacy of a construct relies on generating a richer theoretical structure that is the precondition for predicting observational phenomena, which might not be dealt with by other theories. We need not examine if a construct is true or false, instead, we should focus on whether the construct leads to better understanding of the value and significance of nature, society, and life.

Based on the many-worlds interpretation in quantum theory and the physical theory of quantum entanglement, I present in Section 2 the concept of "nousor", which is the smallest and inseparable unit element with information, and which 
can move faster than light. A general nousor contains core information such as purely forms, aims, agencies, and abilities, and so on; if a general nousor has recorded the special information of external objects, it automatically converts into special nousor, which not only retains the core information of general nousor, but also increases special information of concrete motion of matter. All general nousors have the same information; but special nousors may contain different information of external objects.

Not surprisingly, the notion of nousor discussed here is treated philosophically, which might offer a novel way of thinking and a problem-solving paradigm. Sklar emphasizes the profound role played in science by critical thought on methodologies:

I will argue that various kinds of reasoning that we normally think of as philosophical are deeply embedded in the very practice of science. This embedding of philosophy in science can be clearly seen only when one explores in some detail the ways in which empirical data, hypothesis formation, and philosophical critique all interact in the body of science itself (Sklar, 2000: pp. 7-8).

But we should direct more attention to the dependence of any novel and absurd idea adopted on various cultural or social conditions in which science is, usually unconsciously, embedded, as suggested by Feyerabend's classic study on the absurd point of view:

There is no idea, however ancient and absurd that is not capable of improving our knowledge. The whole history of thought is absorbed into science and is used for improving every single theory (Feyerabend, 1982: p. 47).

I show in Section 3 that self consists of body, brain, and flows of nousors, and self structure includes two parts: ex-self and in-self, which can be depicted by the self schema I presented. Ex-self has different function subareas: ex-sensation, ex-memory, ex-display, ex-processing, ex-operation, and body. In-self also has distinct function subareas: in-sensation, in-memory, in-display, in-processing, inoperation, and body. Discrimination is a common connection area connecting the two parts of self. Combining with the self theory I offered, I suggested that it is the circulatory flow of special nousors in self that forms self-consciousness.

Utilizing the theories of nousor and self, I propose a new view on the origin and meaning of déjà vu, dream, sleepwalking, which would provide empirical verification of the many-worlds interpretation. Counterparts in different reality worlds could communicate with each other through special nousor containing the information packets, releasing into and emerging from the dream, and the dream is a window revealing different worlds. "I" in this world could dream of another "me" in another world, and vice versa; thus the dream offers a channel to understanding each other among different worlds. Otherwise other worlds would be meaningless to us. The highest principle of science and philosophy is determined by the meaning, which is the truth.

I provide details to explain how the cases chosen to illustrate my counterpart (homologous) theory and make the notion of nousor plausible, which might raise critical concerns within methodology, epistemology, psychology, and the- 
ology.

I present in Section 4 the rules followed by different reality worlds, namely, the correspondence rule, the convergence rule, the limitation rule, and the lovehate rule. These rules are permanent and cannot be violated by all worlds. The discussion is concluded in Section 5.

Just as philosopher Popper says, the nature of science is conjecture and refutation. Although the thought in the paper is a little bolder, but the model introducing from it can solve some difficult problems in psychology. The relations between the quantum mechanics and psychology has not been thoroughly disclosed now, but any conjecture about it may be helpful to arouse interesting and curiosity.

\section{Empirical Intermediary among Many-Worlds}

The double-slit experiment (see Brukner \& Zeilinger, 2002; Donati, Missiroli, \& Pozzi, 1973) demonstrates the intriguing interference phenomenon of light and matter particles. Many researchers after Schrödinger offered alternative interpretations: the many-worlds interpretation (1956/1973, 1957; see also Osnaghi, Freitas, \& Freire Jr., 2009; Rubin, 2001, 2005; Deutsch, 1997). In the manyworlds interpretation consciousness plays a less critical role than that in Copenhagen interpretation since here the observer and the observed system are entangled, forming an integrated quantum system. The state of one subsystem is correlated with that of the other subsystem, and quantum decoherence leads to the splitting of many worlds. For the integrated system, the interaction between the observed and observing subsystems causes the total wave function to decompose, and all possible states really exist in different branches. Therefore, each branch of the many worlds represents a reality (DeWitt, 1970, 1971; Wallace, 2003), in contrast to the Copenhagen interpretation in which only one branch is real and exists. Furthermore, each consciousness of reality is aware of the existence of its corresponding consciousness in other realities, via theory and evidence, because there is physical contact among all branches via interference. Here I argue that the many-worlds theory could be further developed to reveal its empirical implications, which are of great value not only to science, but also to philosophy, theology, and society.

Why do we say there is physical contact among all branches via interference? Why would you be able to perceive the existence of consciousness in other worlds? In order to answer these questions, I will present the concept of "nousor", which is derived from the physical phenomenon of quantum entanglement that occurs when pairs or groups of particles are generated or interact in ways such that the quantum state of each particle cannot be fully described without considering the other(s). Measuring the physical properties, such as position, momentum, spin, polarization, etc., of entangled particles, the set of all results are found to be appropriately correlated. One example of entanglement is the double-slit experiment mentioned above; another is a thought experiment discussed by Einstein et al. (1935), in a joint paper with Einstein, Podolsky and Ro- 
sen (1935), describing what came to be known as Einstein-Podolsky-Rosen paradox (EPR paradox), Erwin Schrödinger contributed to this idea shortly thereafter (Schrödinger \& Born, 1935; Schrödinger \& Dirac, 1936).

The thought experiment is briefly as follows. Imagine a two-particle system of zero spin, in which particle 1 is sent to location A, particle 2 is sent to location $B$. Measurements of spin are performed, along axes that are independently chosen, by a Stern-Gerlach device at location A. Particle 1 is measured to be spin up on some axis, particle 2, when measured on the same axis, is always found to be spin down (Figure 1). While particle 1 is traveling, if the axis is changed, the behavior of particle 2 will be found to be also changed (Figure 2). The scheme for measurements on particles looks very similar: the quantum state is different but has very similar properties.

The thought experiment attempted to demonstrate that the quantum mechanical description of physical reality given by wave functions is incomplete. According to the nature of quantum measurement, the behavior of particles leads to effects that can appear paradoxical: it appears that particle 2 of the entangled pair "knows" what measurement has been performed on particle 1 and with what outcome-even though there is no means for such information to be communicated between the particles, which, at the time of measurement, may be separated by arbitrarily large distances. Einstein and others considered such behavior to be impossible, because it seemed to violate the speed limit on the transmission of information in the theory of relativity. Schrödinger is the first person who used the word entanglement to describe the correlation between two particles that interact and then separate, as in the EPR experiment. Like Einstein, Schrödinger was dissatisfied with the concept of entanglement, which was later famously derided, by Einstein, as "spooky action at a distance" (Einstein \& Born, 1971; Kumar, 2011).

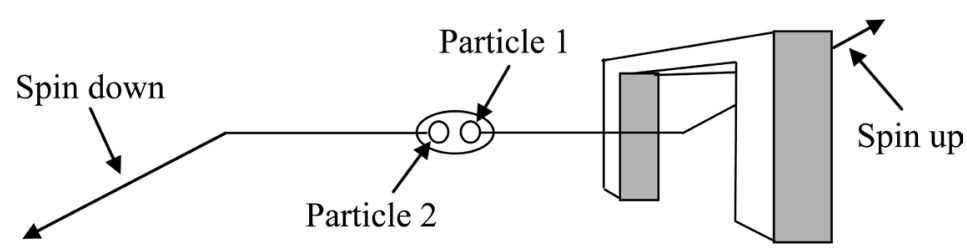

B

A

Figure 1. Two-particle system of zero spin, spin up, spin down. Source: Zukav, 2010, figure 68.

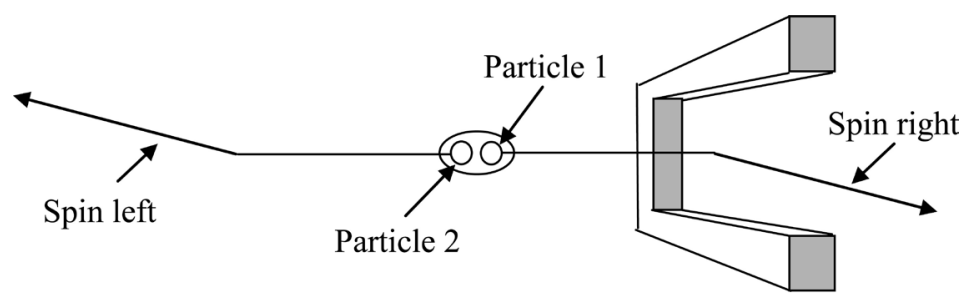

B

A

Figure 2. Two-particle system of zero spin, spin left, spin right. Source: Zukav, 2010, figure 69 . 
A possible resolution to the paradox might be to assume that the state of the particles contains some local hidden variables, whose values effectively determine, right from the moment of separation, the outcomes of the spin measurements. This means that each particle carries all the required information with it, and, at the time of measurement, nothing needs to be transmitted from one particle to the other. Einstein and others believed that, in this way, a more-complete theory might one day be discovered. The resolution from the EPR paper depends on two assumptions, that is: 1) realism-that microscopic objects have real properties determining the outcomes of quantum mechanical measurements; and 2) locality-that reality in one location is not influenced by measurements performed simultaneously at a distant location. More simply, realism meant the moon is there even when not being observed, and locality meant no instantaneous action at a distance.

The seemingly reasonable assumptions, however, were hotly debated within the physics community, most notably between Einstein and Niels Bohr (Kumar, 2011). But the major flaw in EPR's argument was not discovered until 1964, when John Stewart Bell proved that one of their key assumptions, the locality, was inconsistent with the hidden variables interpretation of quantum theory that EPR intended to establish. Specifically, he was able to derive from the two assumptions an important result, namely Bell's inequality, in which he demonstrated an upper limit regarding the strength of correlations that can be produced in any theory obeying local realism, and showed the quantum theory predicts violating this limit for certain entangled systems. Thus, he implied that at least one of the assumptions must be false (Bell, 1964).

Statistically, if the local realism or hidden variables view were correct, then the results would always satisfy Bell's inequality, which was, in part, able to be experimentally tested. However, numerous experiments have shown that Bell's inequality is not satisfied. The earlier experiments were performed by Freedman and Clauser (1972), and Aspect, Grangier and Roger (1982). Recently, some independent experiments have proved that two particles were always entangled after they had been measured (Ma, Zotter, Kofler, Ursin, Jennewein, Brukner, \& Zeilinger, 2012), and demonstrated that the nonlocality of quantum mechanics does not apply only to particles with spacelike separation, but also to particles with temporal separation (Megidish, Halevy, Shacham, Dvir, Dovrat, \& Eisenberg, 2013); Yin (2013) claimed that the speed of Einstein's "spooky action at a distance" is at least 10,000 times fast than light. These studies confirm that, although a loophole or two are still outstanding, the original formulation of quantum mechanics is indeed correct. Therefore, Bell's inequality has been highly commended by a number of scientists, particle physicist Henry Stapp (1975, p. 271) called it "the most profound idea in science".

Bell's work leads to the possibility of exploring seemingly philosophical questions: Why does one particle of the entangled pair "know" what measurement has been performed on another particle? If there is something that can travel faster than the speed of light, then what it would be? 
Our reality world is considered to be a system of four-dimensional space-time, comprising three dimensions of space and one of time, where we cannot directly observe anything that travels faster than light. However, if there is superluminal thing, then its effects could be found among things traveling at or below light speed, and we could analyze the things by our reasoning skills. The latest achievements of quantum mechanics seem to prove the existence of superluminal things, which I name nousor. One of the most important features of nousor is its status of superluminal speeds, that is, nousor can break through the restriction of fourdimension of space-time, enter the higher dimensions, so it cannot be observed by those living in four-dimensional space-time.

What then is nousor? What is nousor's relationship between matter and consciousness? Nousor, in my opinion, is not matter, but an essential component of matter, matter that is a form of interaction between material and nousor. So where there is matter, there is nousor. Nousor is not consciousness, but the combination of nousors releasing their characteristics and functions and special matter with complex structures leads to mass movements of nousors that show consciousness. If the superluminal phenomenon is the evidence of the existence of nousor, so is consciousness. The connection between superluminal and consciousness phenomena is determined by the characteristics and functions of nousor.

I posit that nousor is the smallest and inseparable unit element containing information. Although a fuller taxonomy should be developed later, I can roughly divide nousors into two types: 1) general nousors - those nousors which, similar to Descartes's “natural light” (Descartes, 1985, Med, 3, AT 7:38), or "great light in the intellect" (Descartes, 1985, Med, 4, AT 7:59), contain such core information as a variety of purely forms, aims, agencies, and abilities, and so on, information that combines with materials, and becomes matter; 2) special nousor-when a general nousor is recording, storing, and transmitting the special information of motion of matter, it automatically becomes special nousor, which not only retains all information of general nousor, but also increases special information of concrete motion of matter. The information of general nousors may be mothballed in the special nousor, but sometimes just because a little general information is released from the general nousor, the potential cognitive ability becomes the obvious, and special information is formed. A special nousor does not have other special nousors as constituents, but the information in a special nousor could have other information in other special nousors as constituents. All general nousors are the same, because they have the same information; but special nousors vary greatly since they may contain information concerning the motion of matter.

Consider a pair of entangled particles that are far apart, each of which contains general nousors that will record the behaviors of the particle and convert into special nousors. The information of a special nousor is encrypted for transmission by one of the particles, and its counterpart can decrypt the received information and reform its own behavior by the information. Special nousors' in- 
formation communications within a single object forms microcirculations of special nousors, which work within three dimensional spaces; while special nousors' information communications between a pair of particles forms large circulations of special nousors, which move beyond the restriction of three dimensional spaces. When the internal structure and function of a single object become elaborate or complicated enough, making the circulatory system of special nousors in the single object sufficiently sophisticated and rich, life and consciousness will appear. Unlike special nousors, general nousors that are neither emitted nor received by any single object just permeate the world of matter and convert into special nousors when needed. It can be inferred that general nousors determine the unity, regularity, and hierarchy of the physical and living world, and special nousors determine the otherness, diversity, and richness of the physical and living world.

If there exist other worlds different from each other as well as correlated to each other, and if nousors do exist, then we can presume that our dreams could reveal other reality worlds through nousors. One could sense one's counterparts in other worlds through nousors, and each counterpart could also sense the other in this world. Other reality worlds might appear in our dreams, but we don't realize that they might be as true as the world we are in. We are used to our dreams, not aware that dreams might reflect other worlds in which we are not present. It has been suggested that our dreams merely reflect our world; the dream is not real, but represents various combinations of past experience and memory fragments in our brains. This interpretation views the universe where we are living as an entirety.

Under special circumstances, the special nousor can transfer information among different referent realities. In daily activities and in experience, self is in the waking state, the body is involved in activities and controlled by the flow of nousors in human brain; on the other hand, the activities and experience are recorded by general nousors and contained in special nouosrs as their special information. It is more likely for self in the resting state to release special information received from its counterparts in other realities, since the body in the self is not involved in activities and the general nousor doesn't need to record more special information. One person's brain can emit information to its counterparts whenever a special nousor is formed and stored in the brain; thus someone in another world may receive information of this person through special nousors.

\section{Interpretation: Déjà Vu, Dream, Sleepwalking}

As a thinking person, I don't know directly whether or not a particle has selfawareness, but I know clearly that I myself have self-awareness, and therefore, presumably, those who can communicate with me also have self-awareness. In fact, it doesn't hurt to admit that a particle has self-awareness, since consciousness might be regarded as a sequence from simple to complex (e.g., from particle to amoeba, and then to Einstein). Most of us might agree that humankind whose self structure with body, brain, and flow of nousors lurks the countless mysteries 
of the universe is at a peak or culminating point of the sequence, namely, is a superlative and most sophisticated form of being in this four-dimensional space-time. The complexity and ingeniousness of consciousness has inseparable relation with the quantity and quality of nousor. The scale of flows of special nousors is determined by the quantity of special nousor within the relatively enclosed environment, while the quality means that how much the information (the natural light) in a general nousor releases intellectual information to us, and how complicated the special nousors' microcirculation is. I hold that awareness is essentially the flows of special nousors, which must rest on material container that relies on general nousor, consequently, matter has an integral relation with consciousness due to the nousor intermediary.

Now that the world split into many worlds, and that each of us has counterparts in different reality worlds, how does a person and his counterparts become one, and interact with his counterparts? My hope is that exploration of the problem of circulation system of nousor in self structure will direct further attention to looking at the profound problems of déjà $\mathrm{Vu}$, dream, sleepwalking, and the like.

Let me begin by claiming that we ought to think of self as consisting of body, brain, and flows of nousors, and, as a consequence, that we ought to divide self into ex-self (external self) and in-self (internal self), which will be depicted by the schema I offer. I think that the human self structure includes two parts: ex-self and in-self. Ex-self has different function subareas: ex-sensation (which includes perception), ex-memory, ex-display, ex-processing, ex-operation, and body. In-self also has distinct function subareas: in-sensation (which includes reception, decryption, encryption, and emission), in-memory, in-display, inprocessing, in-operation, and body. There is a common connection area between ex-self and in-self: discrimination (Figure 3).

I may now turn to briefly illustrate the functions of distinct areas in self.

1) EX-sensation. This area has two main sensory sources: a) feelings about the movement of limbs, such as ambulating, leaping, handshake, and the like; and the activities within the body, such as comfort, pain, nervous, satiety, hunger, and so on; b) feelings about the external environment of the body, such as shape, size, color, sound, taste, sour, sweet, bitter, hot, hard, soft, and so forth. All these feelings are generated by sensory organs (e.g., eyes, ears, nose, and tongue). Whenever a special nousor forms, it is coded by forming order. Let me show how nousors work in this area. To take an example, a general nousor records a feeling (the image of house 1) in ex-sensation, and converts into special nousor (1) which flows to perception area, where there are a large number of special nousors that come from memory areas. The circle route of nouse (1) with the image of house 1 may be: perception $\rightarrow$ discrimination $\rightarrow$ ex-memory $\rightarrow$ ex-display $\rightarrow$ ex-processing $\rightarrow$ ex-memory $\rightarrow$ ex-diplay $\rightarrow$ ex-operation $\rightarrow$ body $\rightarrow$ perception. Therefore, some of special nousors enter perception area for the first time, others have, cognitively, been processed in ex-processing area. It should be noted that perception area is a part of ex-sensation (where consciousness can 


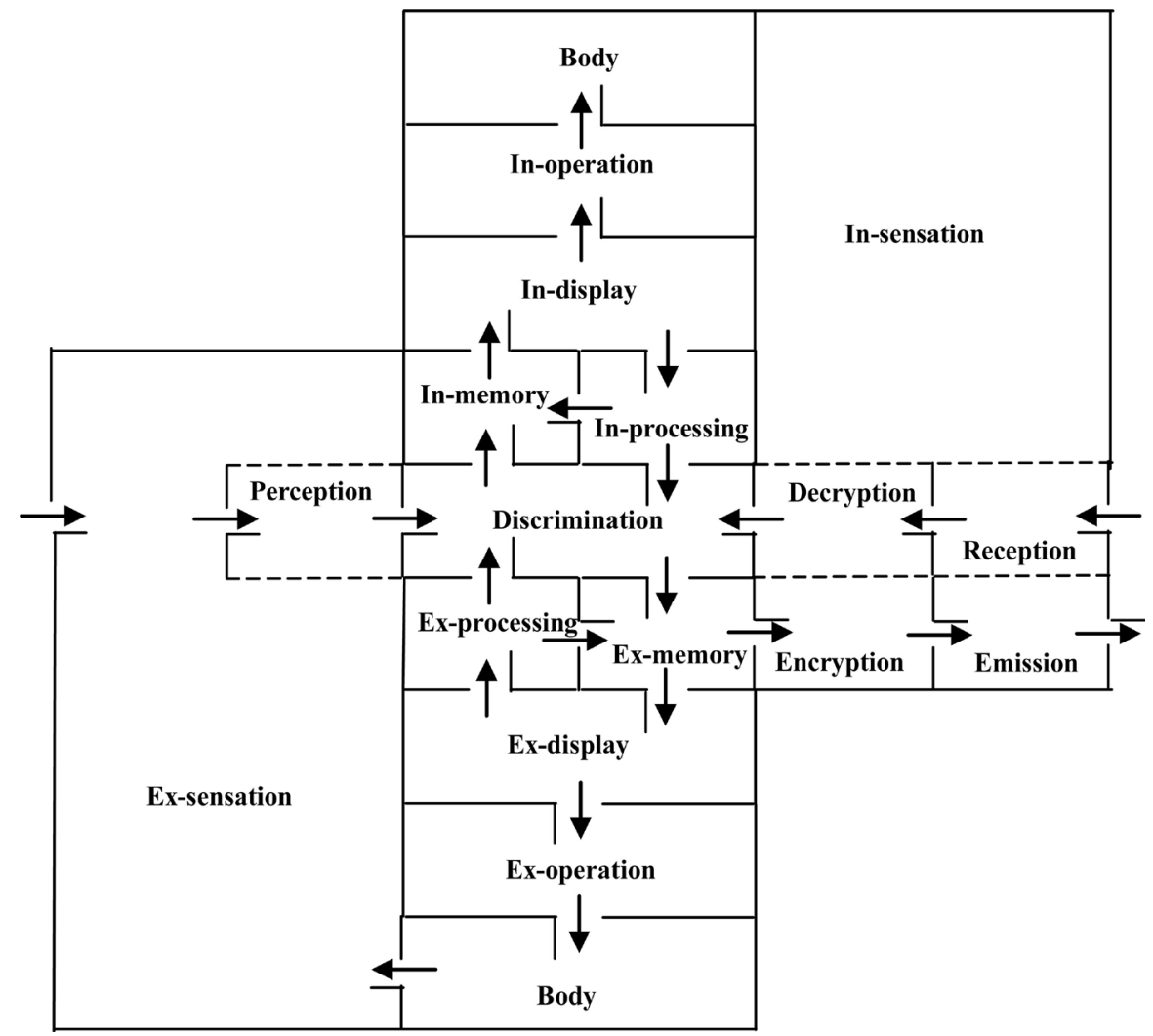

Figure 3. Self structure: ex-self and in-self.

know what it itself is feeling), but different from other part of ex-sensation (where consciousness cannot know what it itself is feeling). For instance, in ex-sensation except for perception area, consciousness can feel an apple's image but cannot know what it is, however, in perception area (where there are numerous special nousors interacting with each other), consciousness can not only feel the apple's image, but also know what it is.

2) Discrimination. The special nousors forming in ex-sensation area are first to enter discrimination area, whose function is to identify the source of special nousors and to shunt them to different areas. If a special nousor comes from exsensation area, it will be sent to ex-memory; if it comes from in-sensation area, it will be sent to in-memory area. Discrimination area sometimes let special nousors from ex-processing area flow into in-memory area, and let those from inprocessing area flow into ex-memory area.

3) Ex-memory. The special nousor that has entered ex-memory occupies a position, never be eliminated. Once a special nousor flows into ex-memory, it will be copied by a general nousor. The same special nousor then flows into encryption area and be encrypted there. After that, the encrypted special nousor flows into emission area, where it is emitted to counterparts. If a special nousor in ex-memory area is motivated by other special nousors, it will be copied by a general nousor, and the same special nousor will flow into ex-display area.

4) Ex-display. If a special nousor flows into ex-display area, it will release special information. For example, when special nousor (1) with the information of 
house 1 enters the ex-display area, the image of house 1 will reappear. This is alleged "recall", or "recollection". The event that special information is released in ex-display area will be recorded by a general nousor, which will convert to a new special nousor involving released information. If a new special nousor with the image of house 1 flows into ex-processing area, into ex-memory area (where it will be copied), and then into ex-display area, one will remember that he had recalled the image of house 1 . The process can unlimitedly be repeated.

5) Ex-operation. If a special nousor in ex-display area carries the information of strong operating instructions (e.g., one lets himself open the window), it will flow into ex-operation area, where it will release instruction information (note, a general nousor in ex-operation area will record this event, and convert to a new special nousor that will flow into ex-memory through body and ex-sensation), generate impulse to act, and thus direct the working of the body. If a special nousor in ex-display area carries the information of weak operating instructions (e.g., one is asked to open the window), it will flow into ex-processing area, where it will interacts with other special nousors, and confirm whether or not to obey the instruction information. If the special nousor confirm to execute instructions, it will combine with some special nousors, become a nousor group, and flow into ex-memory, where the nousor group will be copied, and the same nousor group will flow into ex-display to transmit instruction to open the valve leading to ex-operation, and then into ex-operation. If the special nousor confirm not to execute instructions, it will combine with some other special nousors, become a nousor group, and flow into ex-memory, where the nousor group would not be copied, or would be copied, but not open the valve to ex-operation.

6) Ex-processing. The information of the special nousors entering ex-processing area from ex-display will be analyzed. The processes of dealing with the information in ex-processing area are commonly referred to as "cognitive process", or "cognitive procedure". The differences between higher self and lower self are mainly manifested in ex-processing area. The possible working modes of the nousors in this area should be further studied to reveal the cognitive procedure.

Those special nousors that have been processed in ex-processing area will flow into ex-memory area and be stored. Again, a general nousor can record any activities of a special nousor in ex-processing area and become a new special nousor, which will also flow into ex-memory area and be stored. Some of the special nousors in ex-processing area, if needed, will flow, through discrimination area, into in-memory area.

7) In-sensation. So called "sixth sense" actually refers to in-sensation. A person's in-sensation area is directly relevant to his counterparts. In-sensation area consists of four subareas: encryption, emission, reception, and decryption. Whenever a special nousor is stored in ex-memory area, it will be reproduced by a general nousor. Then the same special nousor will enter into encryption area and be encrypted, and go to emission area to be sent to the counterparts. When a special nousor coming from the counterpart is received in reception area, it will flow into decryption area and be decrypted. If the decryption operation is ac- 
complished successfully, the special nousor will flow into discrimination area; if not, the special nousor will not enter into discrimination area. In general, a person and his counterparts can decrypt the information each other, because they are "one person" with deepest love, and love is the best key to open the code lock. The system of encryption and decryption in in-sensation can ensure that the reception and emission of information take aim at particular objectives.

8) In-memory. The information in in-memory area has two sources: one is the special nousors that are decrypted in decryption area, and that flow, via discrimination area, into in-memory area; the other is the special nousors that generate directly in ex-sensation or discrimination area, and that flows, through discrimination area, into in-memory area. All information coming from the counterparts are stored in in-memory area. Like the special nousors in ex-memroy area, the special nousors in in-memory area will occupy a position, never be deleted. In the cases where all valves of ex-self are largely closed, some of special nousors coming from the counterparts in in-memory area may enter into in-display area; and in the cases where all valves of ex-self are nearly opened, certain special nousors coming from ex-processing area can also flow into indisplay area.

9) In-display. If a special nousor in in-memory area is copied by a general nousor, the same special nousor will form and enter into in-display area, where the information in it will be released. When ex-self is awake (i.e., all valves of exself are largely turned on), the information may be image, sound, or emotional impulse; when ex-self is confusion (i.e., all valves of ex-self are essentially turned off), the information may be dreams, some of which might carry instruction information that unlocks the valves leading to body, turning the dream into sleepwalking. In the case where ex-self is awake, ex-self do not necessarily disturb all activities of in-self. Ex-self, however, sometimes wakes up to the activities occurring in in-display area, sometimes not. All activities of a special nousor in in-display area are recorded by a general nousor, which then becomes a new special nousor containing released information. The new special nousor flows normally into in-processing area, or flow in special circumstances into in-operation area.

10) In-processing. The special nousors that come from ex-processing or counterparts in in-display area will flow into in-processing area. Whenever a special nousor flows into in-processing area, the special nousor will combine with other special nousors in the area, creating special nousor pair, special nousor chain, special nousor group, or special nousor net. During the course of the work of inprocessing (e.g., music or poetic creation), numerous special nousors from exprocessing might flow, via discrimination area, into in-memory area, then into in-processing area. In this process, certain special nousor in in-processing area may release general information, leading to inspiration or insight. In this process, the special nousors coming from counterparts might combine with other special nousors in in-processing area, but ex-self or in-self cannot be aware of it. Any activity in in-processing area will be recorded by a general 
nousor, creating a new special nousor that will be copied by another general nousor. One special nousor will flow, through discrimination area, into exmemory area, and another identical special nousor will enter directly into inmemory area.

11) In-operation. The special nousor that has entered into in-operation area might release instruction information, unlock the valve to body, flow into body to manipulate body. It is the special nousor in in-operation that causes so called "unconscious action" or "habitual action" happening under the circumstance of waking ex-consciousness, or sleepwalking occurring in case where ex-consciousness largely loses. A person's limbs' activities controlled by the special nousors in his in-processing area nearly keep in step with those of his counterparts, and when these activities occur, ex-self cannot perceive them.

12) Body. A general nousor can record the information of muscle operation and body movement within the body (e.g., the whole routine of opening a window), turn into a special nousor, which then flow into ex-memory via ex-sensation and discrimination areas. The information in the special nousor will preserve in ex-memory area forever even if it is not detected by ex-sensation. Any special nousor that has entered into ex-memory will be copied by a general nousor, and will flow into encryption area to be encrypted, and then into emission area to be emitted. The information that cannot be detected and recorded by ex-sensation, however, cannot enter into ex-display area from ex-memory area, nor can it form memories or recollections. In in-operation area, once the valve to body is opened, the body will take action which ex-self cannot intervene. For example, ex-self cannot control such actions as swaying to the music spontaneously, being moved to tears, sleepwalking, and the like. If such unconscious actions fail to be recorded by general nousors in ex-sensation, they will not be recalled. Generally speaking, a sleepwalker cannot remember what happened during his sleepwalking.

It should be noted that ex-self and in-self are two aspects of self, each with functional tilts. Ex-self has closer relations with the world around self, and in exself the ex-consciousness with more initiative can clearly perceive and rationally control limbs' activities; while in-self has closer ties with counterpart worlds, which effect on us in this world mainly through in-self. The in-consciousness in in-self has more passivity, seeming to wait for an unconscious appearance. Exself can sometimes recall afterward, but cannot instantaneously perceive, body's activities in in-self. In the case where ex-self can instantaneously perceive body' actions, if ex-consciousness constraints on the actions, the passivity of the actions would become smaller, and in-consciousness more inert; if ex-consciousness does not interfere with the actions, the passivity of the actions would appear to be greater, and in-consciousness more active.

Naturally, ex-self and in-self are sometimes well coordinated. For instance, the special nousors in ex-self can flow into in-self, and inspire the activity of in-self; while the special nousors in in-self can enter into ex-self and release their information. The flow of special nousors has not only small circular motion in ex-self 
and in-self respectively, but also big circular motion connecting between ex-self and in-self. Again, the flow of special nousors joining a person with one of his counterparts makes up the largest circulation beyond our four-dimensional spacetime.

Thus far I have portrayed what is self and how the flow of special nousors works in self. By using this self schema, I may now try to explain how déjà vu, dream, and sleepwalking come out.

Déjà $v u$. The déjà vu phenomenon is that someone suddenly feels he has experienced what he was doing, an event or a scene reproduced more than once. People often have this feeling that what they are doing seem to happen in reality. Déjà vu pops up, if and only if two special nousors with identical information meet in discrimination area, and the pair comes from ex-sensation and in-sensation area respectively. For example, when special nousor (1) from ex-sensation area runs, in discrimination area, across another special nousor (1) from in-sensation area, the identical information in the two special nousors will be set free, generating so called "déjà vu" phenomenon. Whenever a déjà vu generates in discrimination area, a general nousor will record the event, converting into special nousor (2). In discrimination area, the special nousor (1) from ex-sensation area will flow into ex-memory area, and the other identical nousor (1) from in-sensation area will flow into in-memory area, where the data in nousors (1) will be forever stored. In ex-memory area, if special nousor (1) is excited by other special nousors, it will flow into ex-display area, and then the ex-consciousness could remember the content of the déjà vu; if special nousor (2) is motivated by other special nousors, it will enter into ex-display area, and then the ex-consciousness could remember that he had experienced the déjà vu (Figure 4).

In the recall of a memory, a general nousor in ex-display area will record the event, and produce a new special nousor, which will flow, through ex-processing area, into ex-memory area, and be sent, via in-sensation area, to the counterparts (omitted in Figure 4). If (1) from ex-sensation area and (2) from discrimination area flow into ex-processing area, where they interact with other special nousors, it can be said that ex-consciousness tries to understand the two special nousors. The event happening at ex-processing area will be recorded by a general nousor, producing special nousor (3), which carries not only the information of (1) and (2), but also the information of the interaction among (1), (2), and other special nousors. Once (3) is formed, it will flow into ex-memory area and be stored. Any special nousor stored in ex-memory area will be reproduced by a general nousor, generating an identical special nousor, which will flow, through the encryption area, into emission area, where it will be sent to the counterparts of the self (Figure 4). If special nousor (3) in ex-memory area is reproduced by a general nousor and form an identical special nousor, and if the special nousor enters into ex-display area, then ex-consciousness would remember that he had remembered the déjà vu (omitted in Figure 4). If needed by in-consciousness, (1) (originally from ex-sensation area) and (2) (originally from discrimination 


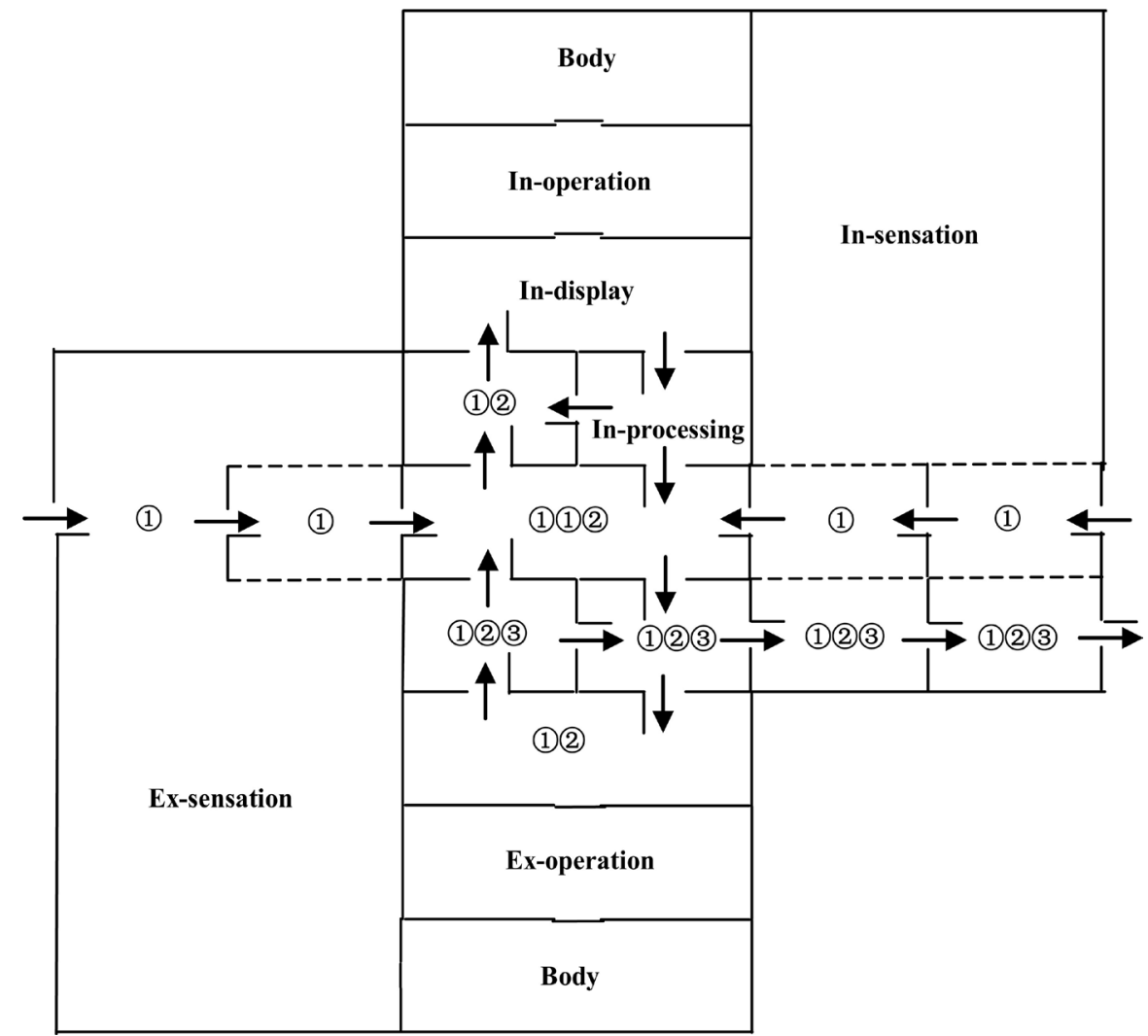

Figure 4. Déjà vu in self.

area) in ex-processing area would flow, through discrimination area, into in-memory area, being reserved. When déjà vu pops up, the valves to ex/ in-operation area and to body are all closed, and the body is instantly in a static state.

Dream. A dream appears, if and only if two or more identical special nousors from different counterparts enter into in-memory area and are merged into one identical special nousor, which opens the valve to in-display area and release its information there. For example, in-sensation receives two identical special nousors, (1) and (1) (coming from different counterparts), which is decrypted in decryption area and flows into discrimination area, and into in-memory area, where a special nousor, (1), will be stored forever, and where other identical special nousors will be consolidated into one, (1), which flows into in-display to release information, forming alleged "dream." The process of the dream will be recorded by a general nousor in in-display area, producing special nousor (2), which carries not only the information of the content of the dream, but also the information of "The dream had formed." After special nousor (2) enters into inprocessing area, it may combine with other special nousors there, and this combination event will be recorded by the general nousors there. Then, special nousor (2) flows, via discrimination area, into ex-memory area, where a general nousor reproduces an identical special nousor (2), which flows into encryption area and is encrypted, and then into emission area to be sent to the counterparts. If a general nousor ex-memory area replicates special nousor (2), which flows 
into ex-display area to release the information, then ex-consciousness could know that he had had a dream, and know the content of the dream, that is, the dream is recalled by ex-consciousness. If special nousor (1) releasing the information in in-display area carries the information of a dream from a counterpart, the dreamer in this world would find that he has a dream in the dream, forming so called "dream within dream" (Figure 5). Put another way, your counterpart Xc in world $\mathrm{c}$ emitted a special nousor to your counterpart $\mathrm{Xb}$ in world $\mathrm{b}$, and $\mathrm{Xb}$ received the special nousor in dream. $\mathrm{Xb}$ then emitted a special nousor containing the information of the dream to you when you were sleeping in world a. It is why the dream within a dream is formed.

When a dreamer's dream happens, all the valves in the ex-sensation area are largely closed, entering too little information from the world where the dreamer is dreaming. Therefore, the dreamer cannot know what is happening around him. Again, during dreaming, the valve connecting ex/in-display area and ex/inoperation area, and the valve connecting ex/in-operation area and body are essentially turned off, so there has no higher range of body movement, except such pretty actions as moving eyeballs, turning over, and so forth. It should be noted that the more special nousors with identical information from in-sensation area, the easier the valve leading to in-display area is opened, and the more clearly the dream occurs and is recalled.

I note that a dream is not imagination, which comes from human beings in the quiescent state or in the waking state (the valves leading to ex-sensation area,

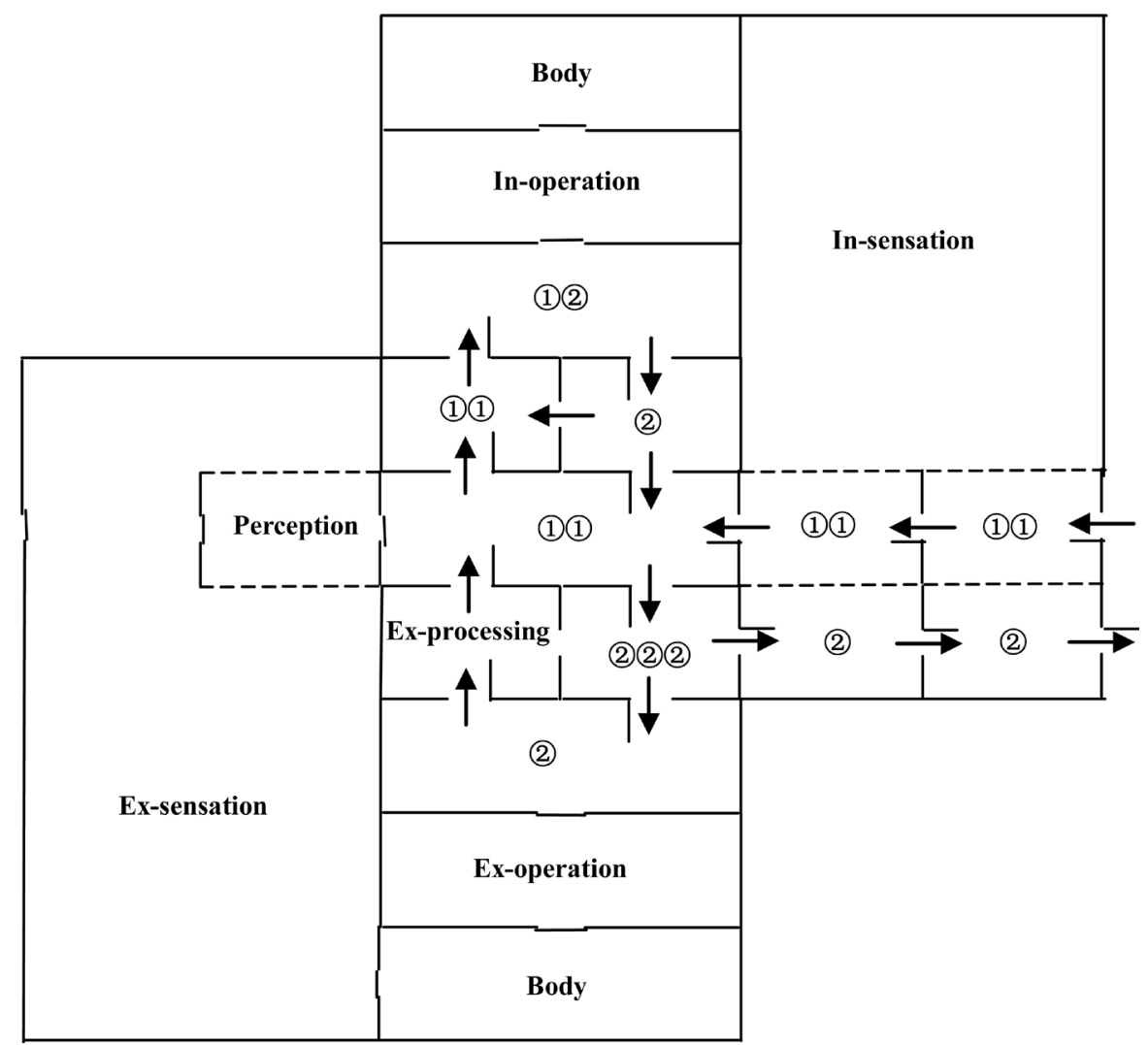

Figure 5. Dream in self. 
to perception area, and to discrimination area are open), while the emergence of dream occurs in the dormant state (the valve connecting to ex-sensation area, to perception area, or to discrimination area is closed). Hence imagination consists of controllable special nousors' circular motion in in-self, in contrast to a dream. Imagination can be considered as a kind of interaction among special nousors, resulting in special nousor pair, special nousor chain, special nousor group, or special nousor net in in-processing area, and the cycle path of the special nousors with particular images may be thus: in-memory $\rightarrow$ in-display $\rightarrow$ inprocessing $\rightarrow$ discrimination $\rightarrow$ in-memory. Again, one would never regard his imagination as experience, because all special nousors from ex-sensation area have been coded and stored in ex-memory area, and some special nousors in inmemory (others from in-sensation area) coming from ex-memory (via ex-display, ex-processing, and discrimination) have been recorded. An individual could not accept the information from his counterpart's imagination, because a special nousor only transmits the information of personal experience from natural processes. The information is transmitted among counterparts, and one person doesn't send out the information of non-experience eventually to himself, let alone prevaricate. A dream, on the other hand, contains real-life information from counterparts in other worlds, but one would never regard his dream as experience either. After one's life turns into the non-material form, however, those dreams will be incorporated into a capsule, becoming first-hand experiences.

Sleepwalking. A person suffers from sleepwalking, if and only if two or more identical special nousors (with the same information) from his different counterparts enter, after being decrypted in depcryption area, into discrimination area, and then into in-memory area; and the environmental information in the special nousors is so identical with or similar to the surroundings during that person's sleep that the special nousor (a copied one) can not only release information in in-display area, but also open the valves to in-operation area and to body. Let me try to describe the process in detail. Imagine in-sensation receives two identical special nousor, (1) and (1), which come from two different counterparts, and which flow, after being decrypted in decryption area, into discrimination area, and then into in-memory area, where the two special nousors are stored, and where a general nousor copies one of them, becoming an identical special nousor, (1), which then flows into in-display area. At this point, if the surrounding environment information in (1) is entirely different from self's external environment, the self has just a dream (as noted before); if it is basically the same as, or identical with, self's external environment, special nousor (1) in in-display area will release the information and form a dream, and simultaneously, release instruction information and open the valves to in-operation area, where it will release instruction information again, and open the valve to body and manipulate the body's movement (Figure 6).

During sleepwalking, all valves of ex-self are closed, and the self loses sensation, so the external information cannot enter the ex-sensation area. The body, 


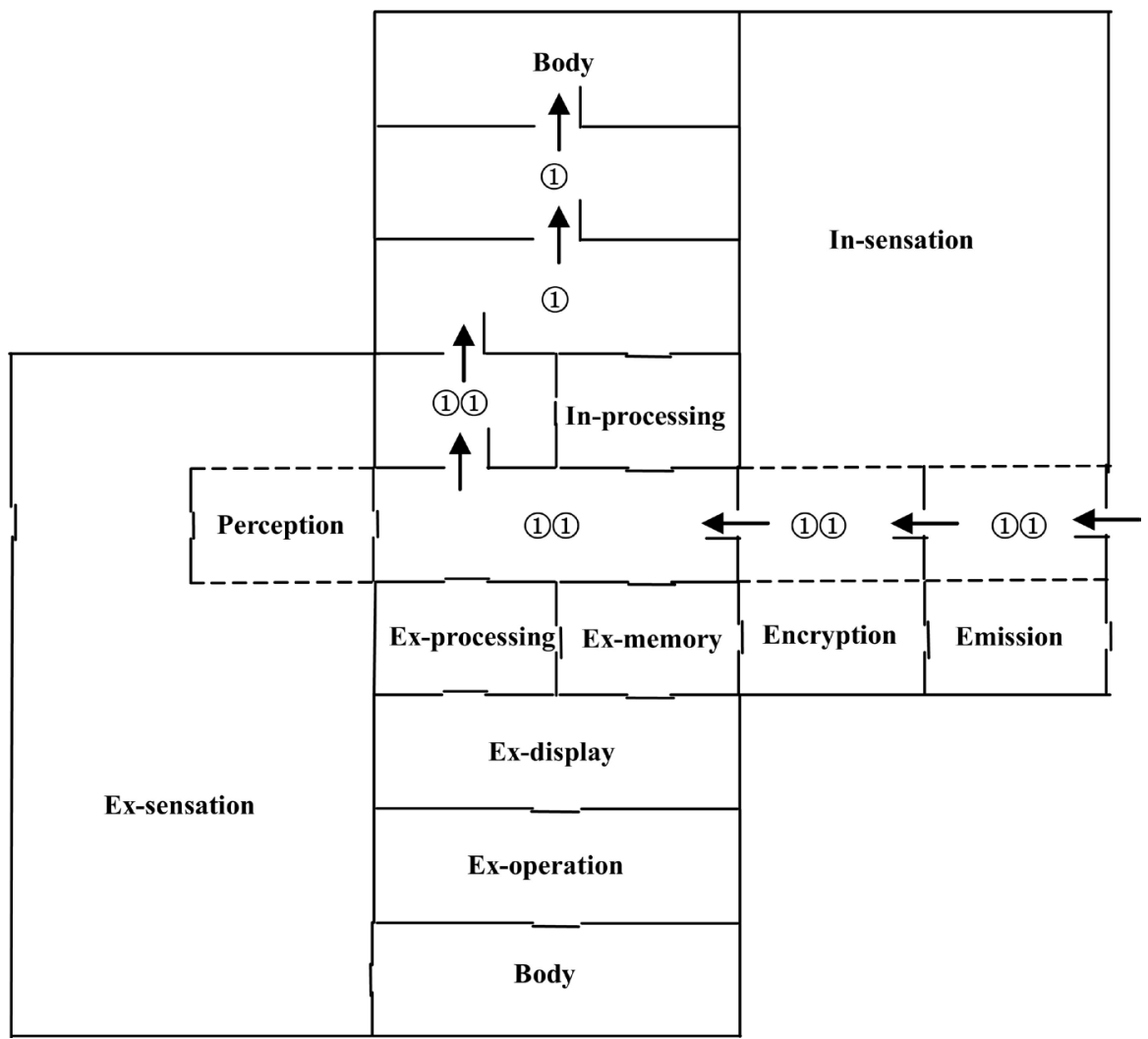

Figure 6. Sleepwalking in self.

however, seems to move or to act in harmony with the surroundings, such as going around obstacles without thinking how to do, picking up a teacup without seeing it, like a puppet being manipulated behind the scenes. In fact, the sleepwalker's five senses do not work, even though they appear to work well together. When special nousor (1) released information in in-display area, a general nousor there may recorded the event and formed a new special nousor, which cannot flow into in-processing area, let alone flow, through discrimination area, into ex-memory area, because the valves along the way were all closed (Figure 6). Therefore, after sleepwalking, the sleepwalker cannot remember that he had experienced the sleepwalking, not to mention knowing the content of the sleepwalking. Moreover, the counterparts of the sleepwalker cannot receive the information from the sleepwalker, since special nousor (1) and the information of the sleepwalking recorded do not stored in ex-memory area, and the valves to encryption and emission area are closed. The more identical special nousors coming from in-sensation area and from different counterparts, the easier the copied special nousor in in-display area opens the valves to in-operation and to body, and more massive or dramatic the strength and amplitude of the sleepwalking has. Sleepwalking is purely a type of the movement of in-self, without the involvement of ex-self.

\section{Rules Followed by Different Reality Worlds}

The world is not "flat" and "single layer", but "stereoscopic" and "many layers". 
The whole reality world consists of many material worlds (reality worlds), which are connected together by nousor (Figure 7, outer circle). One person has many counterparts in different worlds showing different aspects. We regard these counterparts as the one person because of the inseparable correlations among them (Figure 7, intermediate circle). The different worlds obey permanent rules, one of which is the correspondence rule, which states that everyone has only one counterpart in another world, exhibiting various aspects of one person, and the probability of having no counterparts is extremely small. The counterparts of a couple in one world are also a couple in another world; if each couple has one child, these children are the "one person". Image there is a couple, Tom and Mary, who have one child. If Tom's counterpart marries with another individual who is not Mary's counterpart, they will have less chance to give birth to children. If all counterparts of Tom/Mary are dead, he/she will stop dreaming, since the one to one correspondence is broken. I call the correspondence rule the first rule of life in the universe, which cannot be violated.

I need to clarify the concept of the "one person" and explain how to determine an individual's counterparts in other worlds. First, I would like to describe the concept of time presented in this article. Here, the present, the past, and the future indicate the relative sense of certain counterpart in a reality world. For example, if an event happened when you were 18 years old, which is identical to another event happening when your counterpart was also 18 years, then you and your counterpart would think that these two events took place at the same time.

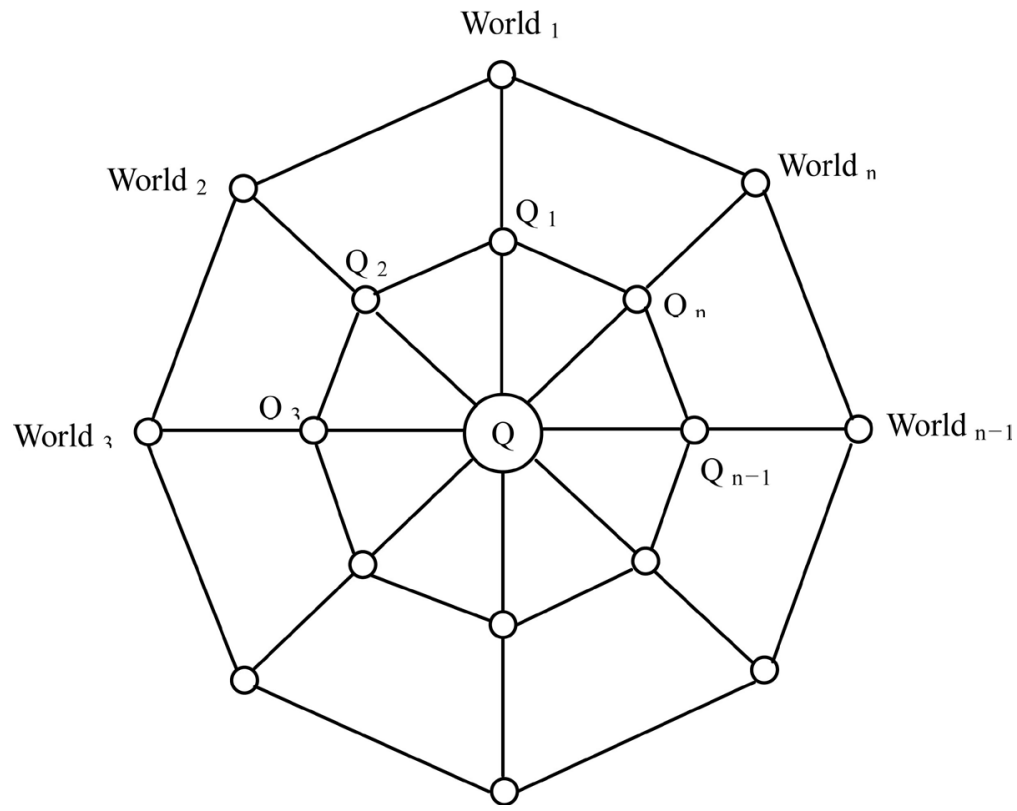

Figure 7. Schematic diagram of counterparts and their worlds. Outer circle: Many worlds are a set of $n$ material worlds (reality worlds), which are connected together by nousor. Intermediate circle: Individual $Q_{n}$ lives in world $n$, and these $n$ persons are counterparts to each other. Each counterpart lives in a separate world, and knows his counterpart's experience through dreams. Inner circle: A whole person $Q$ who doesn't exist until the bodies of $\mathrm{Q}_{1}$ to $\mathrm{Q}_{\mathrm{n}}$ die, and all their souls are capsulated and continue to exist as a spiritual entity. 
This occurrence is just like a video that showed yesterday and is showing again today. A scene in the video that showed yesterday is coincided with a situation in the video playing today, so the two persons in the same scene in the video find that the events happen simultaneously. But if two corresponding pictures in the video are not consistent, the individuals will feel a sense of different time. For instance, if you are 18 years old while you sense that your counterpart is only 8 years old, you will feel the associated event occurs in the past, while your counterpart will feel that an event occurs in the future. On the other hand, if you find that your counterpart is the age of 48 , you will conclude that an event is happening in the future, while your counterpart regards it as a past event. However, most counterparts will feel only present events, i.e., the age you feel in your dream is more likely to be the age your counterpart feels in his dream, because all reality worlds are symmetrical and parallel to each other, and because the special nousor is not affected by time and space.

In reality worlds, the fundamental principle that an individual and all his counterparts are the "one person" doesn't mean the sense of simultaneity, but the one-to-one correspondence determined by the very nature of special nousor. A special nousor transfers information only between a pair of counterparts; anyone in one world can only receive the information from one person (his counterpart) in another world, and anyone in one world can only emit the information to one person in another world. Sometimes you dream some things about other people, which are not directly from those you have dreamed, but from your counterpart who knows them. Though all counterparts originate from one person, they have distinctively different experience in thousands of ways. The role played by the special nousor is to transmit the information between two counterparts, making them have more opportunities to know each other, be aware of each other's existence, perceive each other's passions, and provide help to his counterpart. A pair of counterparts shares the largest interests, since one dies if the other dies, whereas one flourishes if the other flourishes.

It is the nature of the transmission of special nousor that determines, both materially and spiritually, the convergence of a pair of counterparts. A person's father and his counterpart's father also form a pair of counterparts, and as also true for his mother and his counterpart's mother. Furthermore, his spouses, children, brothers and sisters, friends, colleagues, and other surrounding features, all of them and their counterparts in other worlds shall have one-to-one correspondence. There are many material and spiritual similarities, such as body, health, thoughts, knowledge, experience, between a pair of counterparts. Externally they shall also appear to be the same or similar. If one has natural blue eyes or black hair, her counterpart is likely to also have natural blue eyes or black hair. Thus a pair of counterparts shares the same spirit, moral, knowledge, emotion, life, personal relationship, and so on. For example, if Tom is a theist in one world, his counterpart is probably a theist in another world as well; if Mary in one world is well educated, her counterpart is not likely to be illiterate. The convergence of an individual and his counterparts determines their behavior 
disposition. According to the convergence rule, a man wants to become a woman-transsexual because most of the man's counterparts' are woman.

However, in the premise of not violating the first rule of life in the universe, a pair of counterparts might have some interesting differences, some of which lies in interactions with others. If you are unlikely to contact somebody in your world, your counterpart may contact this person in another world. Please note the distinction between "convergence" and "same". Convergence allows some level of variation, with time preceding the variation will not gradually decrease until one person and all her counterparts die, while same means no difference at all among counterparts. All counterparts obey the convergence rule that an individual and all his counterparts are materially and spiritually convergent. I name this the second rule of life in the universe, which shall not be broken.

Using the rule of correspondence and convergence, we can explain some strange dreams. An example comes from my friend: "I had a dream yesterday. The contents of the dream are as follows. My brother and I went to a place, where is the source of a river. We took off our clothes and swam to the opposite bank. When we landed, I found a strange question, that is, why did we still wear clothes, which had been put off on the other side of the river? "

Here I offer a plausible explanation: Two of your counterparts happened to swim with their brothers, one counterpart put off his clothes on this side of the river and swam to the other side, and the other counterpart crossed the river without taking off his clothes. You simultaneously received the two special nousors (information packets) from two counterparts in your dream, and these two scenarios emerged and were superimposed. By the way, if these two counterparts have very similar swimming styles in nearly identical rivers, they are likely to experience déjà vu at the same time.

Moreover, we might derive some falsifiable empirical propositions indirectly from the counterpart theory. For example, it is impossible for any individual to have such a dream in which he becomes someone else, or a bird flying in the sky, or a fish swimming in lake. This is because all reality worlds obey the similar natural laws (although some of them in different reality worlds might seem to be totally different from each other) on the basis of the convergence rule.

In different reality worlds, the one-to-one correspondence between counterparts is not necessarily complete due to the limitations of human beings and the reality world: 1) the number of worlds is finite; 2 ) the population in each world is finite; 3 ) the life span of each person is finite; 4) the total number of a person's counterparts is finite. All of God's creations are finite, and nothing else is infinite except God. I name the rule of limitation as the third rule of life in the universe that could not be broken either. Specifically, I have been learning, growing, and loving in many worlds since I was born. The individual and her counterparts among different reality worlds are always very similar, whether at age 10 or 80 . A person can only live once in one reality world; however, in different real worlds, the life spans of her counterparts are not the same, but convergent. A man dies at 70 in one world, while his counterpart may die at 10 or 90 in other worlds. If a 
person cannot reach the average age in this world, her counterparts are not expected to reach the average age in other worlds because of the convergence effect, while if she has a lifespan over the average in this world, her counterparts are likely to have lifespan longer than average.

If a dead man is dreamed about by others in the same world, he is still alive in another world. A child of my friend died accidentally, and he often has dreams about her in which she is very happy, thus she is still alive in another world. His realization of this will not only help my friend get great comfort, but also change the fate of her counterpart in other worlds, since he can communicate with her counterparts in other worlds by dreams to warn them to avoid certain tragic events.

All counterparts share the same destination eventually, since everyone will die, one after another. The spiritual information of a dead counterpart, all their stories or memories, will be kept in a nonphysical space which obeys the entirely different law from that in reality worlds, being capsulated and continuing to exist as a spiritual entity, who will find that he has accomplished many things that he did not do, and he has repeated same things many times, as if events in his memory become present. A whole person will not be born until the bodies of an individual and all his counterparts are dead, but their souls are still alive (Figure 7 , inner circle). All the information of all these counterparts is contained in one spiritual entity, which will exist independently and eternally, participating no physical interactions. The whole person no long has dreams, since he has no counterpart whose presence is prerequisite for dream. In other words, the whole person is in a mental state similar to a never-ending dream.

A capsule of spirit is not restricted by any material world, so he can reveal truth through direct perception without logic. But the capsule of spirit still obeys the basic rule of love and hate, in which love or hate will ferment, i.e., if love exceeds hatred, love will ferment and imprison hate, while if hatred exceeds love, hatred will ferment and keep love confined. The spiritual entity ruled by love will have happy dreams and never wake up, whereas, the spiritual entity dominated by hatred will have terrible dreams and never wake up. I name the rule of love and hate as the fourth rule of life. A life's true significance lies in the eternal spiritual world, and the short life one spends in real world is only a medium to reflect the immortal spirit. In terms of science and philosophy, the highest principle lies in the meaning, which is the truth. There is no truth without meaning. Our ultimate values lie in faith and love. There is no truth without faith and love.

\section{Conclusion}

Though dream is mysterious and obscure, it helps us understand all aspects of ourselves in different worlds. In the world where we are alive and can dream, we shall constantly gain knowledge and virtue, growing up in love; due to the convergence effect, this healthy condition is likely to be reproduced in other worlds. Then we would have lesser nightmares. Because dreams can reveal the true stories occurring in other worlds, which are expected to take place in our world as 
well, it would be of great value to collect and analyze people's dreams on large scale.

Many parallel worlds are very similar based on the rule of correspondence and convergence, and the dream is the only channel for exchanging information. If there is a major disaster in our counterparts' worlds, similar disaster would take place in our world, and we can receive such information in the dream, which helps us either nip it in the bud or to significantly reduce the loss. However, people haven't made good use of their indispensable dreams.

There are many similar dreams, which are still not considered by scientific communities and relevant government departments. It is of grave importance if the same dreams occur simultaneously in a large population. I thus suggest that governments shall collect their citizen's dreams and use group dreams as a critical indicator to predict catastrophes. Governments should encourage individuals to set up dream centers, build dream databases, and organize research on dream analysis. In this way, we could significantly reduce the loss in future catastrophes.

The nousor theory, the self theory, and the counterpart theory presented in this paper may be opposed by a large majority, not only because the views of these theories are not mainstream views, but also because there are too many questions that need further study. Is nousor the world's origin? What separates nousor from nous, spirit, and soul? How might account for the world's origin, its subject and object, its whole and part, its chief and subordinate? Why do matter and consciousness have different levels? Why does God copy our world? How does the counterpart theory explain other psychological phenomena, such as homosexuality, telepathy, clairvoyance, psychokinesis, demand, cognition, interest, community, evasion, and creativity? It is almost impossible to clearly state all these problems in one article. It is necessary for our colleagues to study together and form a new academic community in the future.

\section{Acknowledgements}

I would like to think Raymond Chester Russ, David Deutsch, and several anonymous referees for their helpful comments and suggestions.

\section{Funding}

This work was supported by Huaqiao University's Research Base for Humanities and Social Sciences under Grants 16SKBS303. Any opinions, findings, and conclusions expressed in this paper are those of the author and do not necessarily reflect the views of Huaqiao University's Research Base for Humanities and Social Sciences.

\section{References}

Aspect, A., Grangier, P., \& Roger, G. (1982). Experimental Realization of Einstein-Podolsky-Rosen-Bohm Gedankenexperiment: A New Violation of Bell's Inequalities. Physical Review Letters, 49, 91-94. https://doi.org/10.1103/PhysRevLett.49.91 
Bell, J. (1964). On the Einstein Podolsky Rosen Paradox. Physics, 1, 195-200.

Brukner, C., \& Zeilinger, A. (2002). Young's Experiment and the Finiteness of Information. Philosophical Transactions of the Royal Society of London, 360, 1061- 1069. https://doi.org/10.1098/rsta.2001.0981

Descartes, R. (1985). The Philosophical Writings of Descartes. Cambridge: Cambridge University Press.

Deutsch, D. (1997). The Fabric of Reality: The Science of Parallel Universes and Its Implications (Chapter 2, Shadows, pp. 32-54). Penguin Books Ltd.

DeWitt, B. S. (1970). Quantum Mechanics and Reality: Could the Solution to the Dilemma of Indeterminism Be a Universe in Which All Possible Outcomes of an Experiment Actually Occur? Physics Today, 23, 30-40. https://doi.org/10.1063/1.3022331

DeWitt, B. S. (1971). Every Quantum Transition Taking Place on Every Star, in Every Galaxy, in Every Remote Corner of the Universe Is Splitting Our Local World on Earth into Myriads of Copies of Itself. Physics Today, 24, 38-44.

Donati, O., Missiroli, G. F., \& Pozzi, G. (1973). An Experiment on Electron Interference. American Journal of Physics, 41, 639-644. https://doi.org/10.1119/1.1987321

Einstein, A., \& Born, M. (1971). Letter from Einstein to Max Born, 3 March 1947. In B. M. Walker (Ed.), The Born-Einstein Letters; Correspondence between Albert Einstein and Max and Hedwig Born from 1916 to 1955 (pp. 157-158). New York.

Einstein, A., Podolsky, B., \& Rosen, N. (1935). Can Quantum-Mechanical Description of Physical Reality Be Considered Complete? Physical Review, 47, 777-780. https://doi.org/10.1103/PhysRev.47.777

Feyerabend, P. (1982). Against Method. Oakland, CA: University of California Press.

Freedman, S. J., \& Clauser, J. F. (1972). Experimental Test of Local Hidden-Variable Theories. Physical Review Letters, 28, 938-941. https://doi.org/10.1103/PhysRevLett.28.938

Henry, S. P. (1975). Bell's Theorem and World Process. Nuovo Cimento, 29B, 270-271.

Kumar, M. (2011). Quantum: Einstein, Bohr, and the Great Debate about the Nature of Reality (Reprint ed., pp. 305-306). W. W. Norton \& Company.

Ma, X. S., Zotter, S., Kofler, J. Ursin, R., Jennewein, T., Brukner, Č., \& Zeilinger, A. (2012). Experimental Delayed-Choice Entanglement Swapping. Nature Physics, 8, 479-484. https://doi.org/10.1038/nphys2294

Megidish, E., Halevy, A., Shacham, T., Dvir, T., Dovrat, L., \& Eisenberg, H. S. (2013) Entanglement Swapping between Photons That Have Never Coexisted. Physical Review Letters, 110, Article ID: 210403.

https://doi.org/10.1103/PhysRevLett.110.210403

Osnaghi, S., Freitas, F., \& Freire, J. O. (2009). The Origin of the Everettian Heresy. Studies in History and Philosophy of Modern Physics, 40, 97-123.

Rubin, M. A. (2001). Locality in the Everett Interpretation of Heisenberg-Picture Quantum Mechanics. Foundations of Physics Letters, 14, 301-322.

https://doi.org/10.1023/A:1012357515678

Rubin, M. A. (2005). There Is No Basis Ambiguity in Everett Quantum Mechanics. Foundations of Physics Letters, 17, 323-341. https://doi.org/10.1023/B:FOPL.0000035668.37005.e0

Schrödinger, E., \& Born, M. (1935). Discussion of Probability Relations between Separated Systems. Mathematical Proceedings of the Cambridge Philosophical Society, 31, 555-563. https://doi.org/10.1017/S0305004100013554 
Schrödinger, E., \& Dirac, P. A. M. (1936). Probability Relations between Separated Systems. Mathematical Proceedings of the Cambridge Philosophical Society, 32, 446-452. https://doi.org/10.1017/S0305004100019137

Sklar, L. (2000). Theory and Truth: Philosophical Critique within Foundational Science. Oxford: Oxford University Press.

Wallace, D. (2003). Everettian Rationality: Defending Deutsch's Approach to Probability in the Everett Interpretation. Studies in History and Philosophy of Modern Physics, 34, 415-438. https://doi.org/10.1016/S1355-2198(03)00036-4

Yin, J. et al. (2013). Bounding the Speed of Spooky Action at a Distance. Physical Review Letters, 110, Article ID: 260407. https://doi.org/10.1103/PhysRevLett.110.260407

Zukav, G. (2010). The Dancing Wu Li Masters: An Overview of the New Physics (Chinese ed., pp. 275-276). Hainan: Hainan Publishing House.

Submit or recommend next manuscript to SCIRP and we will provide best service for you:

Accepting pre-submission inquiries through Email, Facebook, LinkedIn, Twitter, etc. A wide selection of journals (inclusive of 9 subjects, more than 200 journals)

Providing 24-hour high-quality service

User-friendly online submission system

Fair and swift peer-review system

Efficient typesetting and proofreading procedure

Display of the result of downloads and visits, as well as the number of cited articles

Maximum dissemination of your research work

Submit your manuscript at: http://papersubmission.scirp.org/

Or contact ojpp@scirp.org 